\title{
Urinary extracellular vesicular release of aquaporins in patients with renal transplantation
}

\author{
Sayaka Oshikawa-Hori ${ }^{1}$, Naoko Yokota-Ikeda ${ }^{2}$, Hiroko Sonoda ${ }^{1}$ and Masahiro Ikeda ${ }^{1 *}$ (D)
}

\begin{abstract}
Background: Diuresis has been observed within a week following renal transplantation, suggesting that the procedure causes acute disturbance of renal water homeostasis. Aquaporin (AQP) 1 and AQP2, important proteins for renal water reabsorption, have been identified in urinary extracellular vesicles (UEV-AQP1 and -AQP2), and experimental studies have shown that the presence of UEV-AQP1 and -AQP2 may be an indicator of their levels of expression in the kidney. However, the release patterns of UEV-AQP1 and -AQP2 during the acute phase following renal transplantation are largely unknown.
\end{abstract}

Methods: In this study, we examined the release of uEV-AQP1 and -AQP2 in recipients until 6 days (day 6) after renal transplantation. At Miyazaki prefectural Miyazaki Hospital, Japan, uEVs were obtained from 7 recipients, all of whom had received renal allografts from living donors. uEVs were isolated by differential centrifugation.

Results: Immunoblotting analysis showed that the release of UEV-AQP2 was significantly decreased on day 1 in comparison with a control sample (from 3 healthy volunteers), accompanied by high urine output and low urine osmolality. Thereafter, the level increased gradually to the control level by day 6 . The release pattern of uEV-AQP1 was similar to that of UEV-AQP2, but the levels did not reach statistical significance in comparison with the control level at any of the time points examined. Evaluation of the relationship between urinary osmolality and uEV-AQPs revealed a significant correlation for UEV-AQP2, but not for UEV-AQP1.

Conclusion: These results indicate that acute diuresis after renal transplantation might be due to a decrease in the renal expression of AQP2, whose level can be estimated from the amount released in uEVs.

Keywords: Extracellular vesicles, Renal transplantation, Aquaporin-1, Aquaporin-2, Kidney, Urine, Exosomes

\section{Background}

Following the introduction of renal transplantation in the 1950s, diuresis in the recipients had been occasionally observed within a week after the operation [1, 2]. However, the underlying mechanisms had remained largely unknown because the molecular mechanism responsible for urinary concentration had yet to be clarified. In 1992, Agre's group discovered aquaporin-1 (AQP1), which is now known to play critical roles in water reabsorption in the renal proximal tubule and in maintaining the renal medullary osmotic gradient $[3,4]$.

\footnotetext{
* Correspondence: a0d302u@cc.miyazaki-u.ac.jp

'Department of Veterinary Pharmacology, Faculty of Agriculture, University of Miyazaki, Gakuenkibanadai-Nishi 1-1, Miyazaki 889-2192, Japan

Full list of author information is available at the end of the article
}

One year later, Sasaki's group found another AQP, $\mathrm{AQP} 2$, which is regulated by vasopressin, and subsequent studies have revealed that this protein is involved in the mechanism of urinary concentration in collecting ducts $[4,5]$. Currently, thirteen AQPs (AQP0-12) are known to exist in mammals, of which eight (AQP1-4, $6-8,11)$ are expressed in renal epithelial cells [4].

Extracellular vesicles (EVs) are composed of exosomes, microvesicles, and apoptotic bodies, among which exosomes and microvesicles have been a focus of intense translational research with the aim of developing novel biomarkers and therapeutics for renal disease [6-10]. Exosomes, small EVs $(30-150 \mathrm{~nm}$ in diameter), are derived from internal vesicles enclosed in multivesicular bodies (MVBs). When the MVBs fuse with the plasma

(C) The Author(s). 2019 Open Access This article is distributed under the terms of the Creative Commons Attribution 4.0 International License (http://creativecommons.org/licenses/by/4.0/), which permits unrestricted use, distribution, and 
membrane, internal vesicles are released as exosomes into extracellular fluids such as blood and urine. Microvesicles are larger EVs with a diameter of $50-2000 \mathrm{~nm}$, budding directly from the plasma membrane. Because of the structural similarity of exosomes and microvesicles, and the lack of appropriate markers that can discriminate between them, the International Society for Extracellular Vesicles has recommended the use of the generic term "EVs" for all released vesicles $[8,10]$, and we adopted this recommendation in the present study.

EVs are known to selectively contain functional proteins from their cells of origin, and their release has also been observed to depend on the state of their original cells [11-15]. Among the various AQPs, AQP1 and AQP2 have been identified in urinary EVs (uEVs), and several studies with animal models have shown that their levels in $\mathrm{uEVs}$ are related to their levels in the kidney [15-17].

Several experimental studies have shown that AQP1 and AQP2 might be involved in the diuresis evident during the acute phase after renal transplantation [18-20]. On the other hand, information from humans is still very limited. We have observed that the release of AQP1-bearing uEVs (uEV-AQP1) was decreased in a renal transplant recipient $48 \mathrm{~h}$ after surgery [16]. However, to our knowledge, no other information is available, and the release patterns of $\mathrm{uEV}-\mathrm{AQP} 1$ and -AQP2 in the acute phase of renal transplantation in humans remain largely unknown.

In order to characterize the release patterns of uEV-AQP1 and -AQP2 after renal transplantation, we examined seven recipients in the acute phase after surgery. We also measured marker proteins for uEVs including tumor susceptibility gene 101 (TSG101) protein and apoptosis-linked gene 2-interacting protein X (Alix) $[15,21]$.

In the present paper, since uEVs are produced through the above-mentioned mechanisms, we use the term "release" for uEVs such as hormones and autacoids.

\section{Methods}

\section{Study participants}

This study was approved by the Miyazaki Prefectural Miyazaki Hospital Institutional Review Board in accordance with the Ethical Guidelines for Clinical Studies in Japan (Miyazaki of Health, Labour and Welfare, July 30, 2003, Amended December 28, 2004). Spot urine samples were obtained from 7 patients $(2$ males aged 42 and 52 and 5 females aged $25,43,53,59$, and 62 ) on days 1,2 , and 6 after receiving renal allografts from living donors (including one case of pre-emptive renal transplantation) at Miyazaki Prefectural Miyazaki Hospital Institution between 2009 and 2011. All of the patients were treated with a triple immunosuppressive regimen including tacrolimus (0.15-0.3 mg/kg/day, Astellas Pharma Ltd., Tokyo, Japan), mycophenolate mofetil ( $2 \mathrm{~g} /$ body/day, Novartis, Basel, Switzerland), and methylprednisolone (8 mg/body/day, Sanofi Aventis, Paris, France). Also, basiliximab (20 mg/body/day, Novartis) was added on days 0 and 4 .

We employed a standard form of fluid management, including infusion during the operation and compensatory infusion in accordance with urine output after the operation. Since the patients had rather limited water intake during the first $24 \mathrm{~h}$ after the operation, compensatory infusion was carefully performed. Thereafter, their water intake increased. When the patients were able to drink well and their water balance was maintained, the infusion was withdrawn.

A spot urine sample from donor was also collected on days 1, 2, and 6 in some cases. Control spot urine samples were also collected from 3 healthy male volunteers aged 21 to 46 years. A sample made from the three healthy volunteers was always used to normalize quantification in each gel. Urine osmolality was measured using an automatic osmometer (Osmostation om-6060, Arkray, Kyoto, Japan).

\section{Isolation of uEVs}

Isolation of $\mathrm{uEVs}$ was performed as described previously [16]. Briefly, just after collection, each urine sample was mixed with a protease inhibitor mixture (final concentration, $1 \mathrm{mM}$ EDTA, $0.5 \mathrm{mM}$ p-amidinophenyl methanesulfonyl fluoride hydrochloride, and $0.12 \mathrm{mM}$ leupeptin). Thereafter, the urine was centrifuged at 1000 $\mathrm{x} \mathrm{g}$ at $4{ }^{\circ} \mathrm{C}$ for $15 \mathrm{~min}$ to eliminate urinary debris. The supernatant was centrifuged at $17,000 \mathrm{x} g$ at $4{ }^{\circ} \mathrm{C}$ for 30 min. The resulting supernatant was then ultracentrifuged at $200,000 \times \mathrm{g}$ (Optima TL Ultracentrifuge, Beckman Instruments, $\mathrm{CA}$ ) at $4{ }^{\circ} \mathrm{C}$ for $1 \mathrm{~h}$ to isolate a low-density membrane fraction. Many studies use the term exosomes to refer to the EVs in this low-density membrane fraction [10]. After the ultracentrifugation, a protease inhibitor mixture was added to the pellet. Subsequently, the suspended pellet was solubilized in $4 \times$ sample buffer ( $8 \%$ SDS, $50 \%$ glycerol, $250 \mathrm{mM}$ TrisHCL, $0.05 \%$ bromophenol blue, 400 mM DTT, pH 6.8), then incubated at $37^{\circ} \mathrm{C}$ for $30 \mathrm{~min}$ and stored at $-80^{\circ} \mathrm{C}$ until use.

\section{Gel electrophoresis and immunoblot analysis}

Immunoblot analysis was performed as described previously [16]. Briefly, each urine sample was loaded with an equal amount of creatinine per lane, and separated by SDS-PAGE. For the detection of each protein, we used the following primary and secondary antibodies: antiAQP1 (catalog no. sc-20810, Santa Cruz Biotechnology, Santa Cruz, Dallas, TX), anti-AQP2 (catalog no. sc-9882, 
Santa Cruz Biotechnology), anti-TSG101 (catalog no. ab83-100, Abcam, Cambridge, UK), anti-Alix (catalog no. sc-49268, Santa Cruz Biotechnology), anti-rabbit IgG (catalog no. 7074, Cell Signaling Technology, Danvers, MA), anti-mouse IgG (catalog no. 1858413, Thermo Fisher Scientific, Waltham, MA), and anti-goat IgG (catalog no. P0449, Dako Japan, Tokyo, Japan). Antibody-antigen interactions were visualized using the SuperSignal West-Femto Chemiluminescence detection system (Thermo Fisher Scientific, Waltham, MA). Quantitative analysis of the resulting bands was performed using the WinRoof software package version 5.7 (Mitani, Tokyo, Japan).

\section{Statistical analysis}

Box plots were generated using the BoxPlotR: a web tool for generation of box plots (http://boxplot.tyerslab.com) [22]. Differences between renal transplant patients and controls were analyzed by the Mann-Whitney $U$ test or, when the sample mean should be compared with a hypothesized population mean, one sample t-test using EZR (Saitama Medical Center, Jichi Medical University, version 1.29) (http://www.jichi.ac.jp/saitama-sct/SaitamaHP.files/statmedEN.html) on $\mathrm{R}$ commander (version 2.1-7), which is a graphical user interface for $\mathrm{R}$ (The $\mathrm{R}$ Foundation for Statistical Computing, version 3.2.1) [23]. Statistical analysis of correlations between osmolality and uEV-AQPs was performed using Pearson's correlation test. All values were considered to be statistically significant at $P<0.05$.

\section{Results}

Blood parameters in renal transplant recipients at 1 (day 1), 2, and 6 days after surgery are shown in Table 1 . The levels of blood urea nitrogen (BUN) in these patients were within the normal range $(8.6-22.9 \mathrm{mg} / \mathrm{dl})$ at all of the time points examined. The serum creatinine $(\mathrm{SCr})$ concentration on day 1 was higher and then decreased to the normal range (male, $0.6-1.2 \mathrm{mg} / \mathrm{dl}$, female, $0.4-$ $1.0 \mathrm{mg} / \mathrm{dl}$ ). Figure 1 summarizes data for daily urine volume and urine osmolality. Urine output on day 1 was markedly higher than normal $(500-2000 \mathrm{ml} /$ day), and thereafter decreased but still remained high. Similarly, urine osmolality was obviously low in comparison with the normal range $(580-1130 \mathrm{mOsm} / \mathrm{kg} \mathrm{H} 2 \mathrm{O})$ at any of the time points examined, especially on days 1 and 2 . In fact, when we compared the urinary osmolality of the

Table 1 BUN and SCr concentrations in recipients

\begin{tabular}{llll}
\hline & Day 1 & Day 2 & Day 6 \\
\hline BUN $(\mathrm{mg} / \mathrm{dl})$ & $18.6 \pm 4.0(7)$ & $12.2 \pm 2.6$ & $15.1 \pm 3.2$ \\
$\mathrm{SCr}(\mathrm{mg} / \mathrm{dl})$ & $2.6 \pm 0.7(7)$ & $1.2 \pm 0.2$ & $1.0 \pm 0.2$ \\
\hline
\end{tabular}

Values are represented as means \pm SE. Parentheses indicate the numbers of patients patients with our pooled data from healthy volunteers (477-946 mOsm/kg H2O, $n=9$ ), urine osmolality in the recipients was significantly lower at any of the time points examined (unfortunately, urine volume data for healthy volunteers were not available). On the other hand, on days 1 and 2, the water balance was maintained by isotonic infusion (see Methods). These data suggested that the patients we had recruited exhibited acute diuresis after renal transplantation, being most prominent on day 1.

Figure 2 shows a representative immunoblot of uEVproteins in a recipient and a donor. The levels of AQP1 and AQP2 in the recipient were reduced in comparison with those of the control (shown on the far right) on days 1 and 2, while on day 6 the levels recovered to the control level. The level of TSG101 showed alterations similar to those of AQPs, being somewhat higher than the control level on day 6. The level of Alix was decreased on day 2 and increased on day 6 . In the donor, the levels of AQP1 and AQP2 were moderately decreased on day 2. Figure 3 summarizes the data from the immunoblot analyses of the recipients. Release of $\mathrm{uEV}$ AQP2 in recipients was significantly decreased on day 1. On day 2, in 5 out of 7 patients, a decreased release of uEV-AQP2 was observed, but the decrease did not reach significance. The level on day 6 was comparable to the control level. For AQP1, a similar tendency was observed but did not reach statistical significance in comparison with the control level at any of the examined time points. Release of uEV-TSG101 was significantly decreased on day 1 and the level on day 6 tended to be increased. For Alix, the levels were increased at all of the time points examined, becoming statistically significant on days 2 and 6 in comparison with the control level.

Next, we examined the correlation between urine osmolality and release of $\mathrm{uEV}-\mathrm{AQP} 1$ or $-\mathrm{AQP} 2$ using all of the data from recipients (Fig. 4). This revealed a significant relationship between osmolality and release of $u E V-A Q P 2$, but not that of uEV-AQP1.

\section{Discussion}

One major finding in this study was that release of uEVAQP2 might be applicable as a biomarker of the urinary concentration defect in renal transplant recipients. A significant reduction in the release of $\mathrm{uEV}-\mathrm{AQP} 2$ was observed on day 1 , accompanied by high urinary output and low urine osmolality. Thereafter, the release of uEV-AQP2 gradually increased to the control level, along with recovery of the urinary concentration mechanism. Furthermore, a significant correlation between urinary osmolality and release of uEV-AQP2 was observed. On the other hand, the correlation for AQP1 was not significant. These data indicate that release of uEV-AQP2 might be an indicator of the 


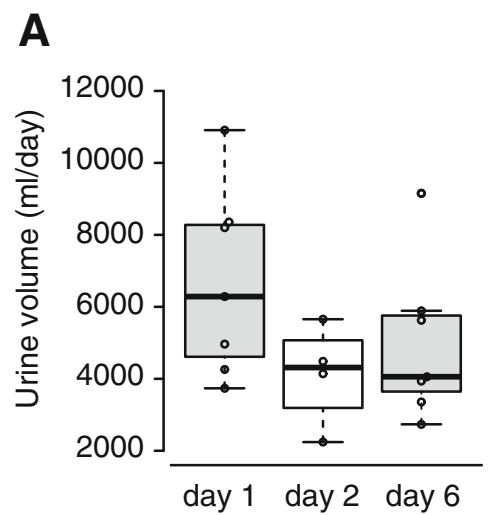

(7)

(4)

(7)
B

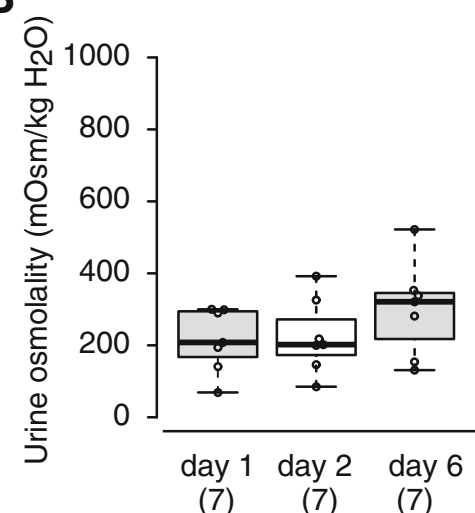

Fig. 1 Urinary volume and osmolality in renal transplant recipients. Dot and box plots of urine volume (a) and osmolality (b) are shown for recipients on days 1, 2, and 6. The central line, top border and bottom border represent the median, 25th and 75th percentiles, and the whiskers show 1.5 times the interquartile range from the lower and upper percentiles. Only four samples were available for measurement of urine volume on day 2. Parentheses indicate the numbers of patients

urinary concentration defect in recipients within a week after renal transplantation.

Previously, we observed a decrease in release of $\mathrm{uEV}$ AQP2 in rats treated with gentamicin [17]. Along with this decrease, we also detected a defect in the urinary concentration mechanism and polyuria, and a reduced level of renal AQP2 expression. Although the present study did not examine the renal expression level of AQP2, our previous observation strongly suggests that the decrease in the release of $u E V-A Q P 2$ in recipients was due to the reduced renal expression of AQP2.

In the present study, tacrolimus was used to suppress the immunoreaction after renal transplantation. Tacrolimus is

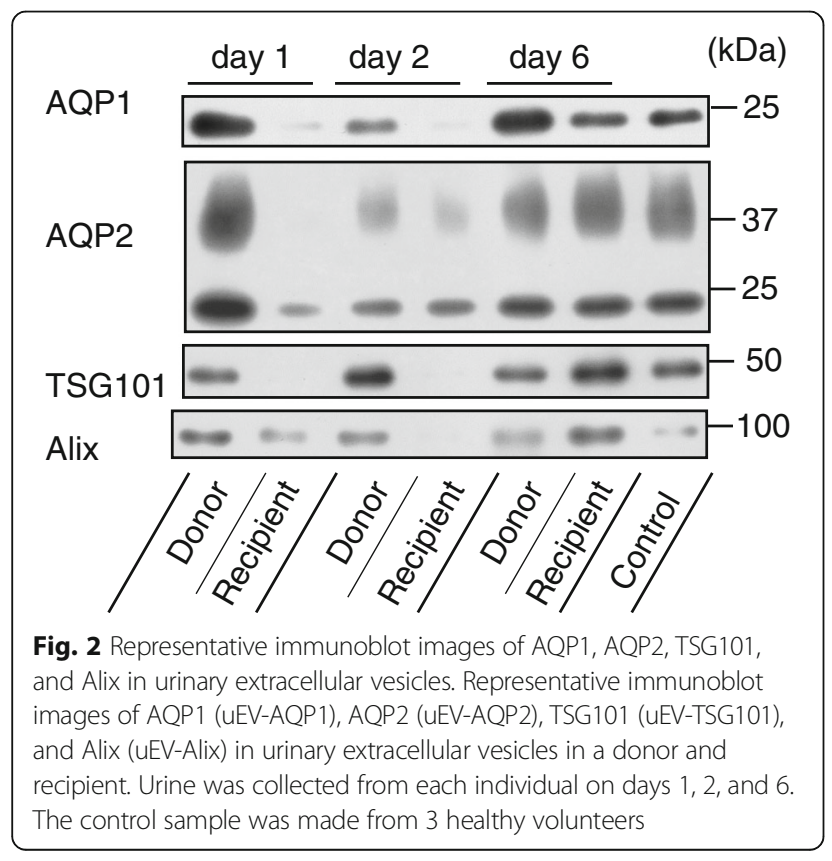

known to inhibit phosphatase $2 \mathrm{~B}$ [24], which would be expected to enhance the phosphorylation of AQP2. Increase in the phosphorylation of AQP2 in the renal collecting duct cells would stimulate the translocation of AQP2 from intracellular vesicles to the apical membrane, and the expression of AQP2 protein through increased transcription [4]. Therefore, treatment of patients with tacrolimus is considered to affect the release of uEV-AQP2. However, several studies have shown that tacrolimus had little effect on both the phosphorylation and the expression of AQP2 in the renal collecting duct cells [24, 25], and thus the contribution of tacrolimus to the release of $\mathrm{uEV}-\mathrm{AQP} 2$ was considered to be minimal.

In contrast to the release of $\mathrm{uEV}-\mathrm{AQP} 2$, that of $\mathrm{uEV}$ AQP1 tended to be decreased on day 1 , but the decrease did not become statistical significant in comparison with the control group. Several experimental studies have revealed that warm renal ischemia-reperfusion dramatically decreases the renal expression of AQP1 [26, 27], suggesting a decrease in the release of uEV-AQP1 after renal ischemia-reperfusion. On the other hand, in the human renal transplantation setting, renal ischemiareflow has been performed at a low temperature in comparison with the above experimental models. It has been reported that a lower temperature reduces the severity of renal ischemia-reperfusion injury [28]. Therefore, a possible reason for the non-significant release of UEV-AQP1 on day 1 may have been the different temperature conditions for renal ischemia-reperfusion between humans and experimental animals.

In the present study, the release patterns of uEVTSG101 and -Alix, both of which have been used as exosomal marker proteins $[15,21]$, did not show a comparable tendency. The release of uEV-TSG101 was significantly reduced on day 1 and then increased to 


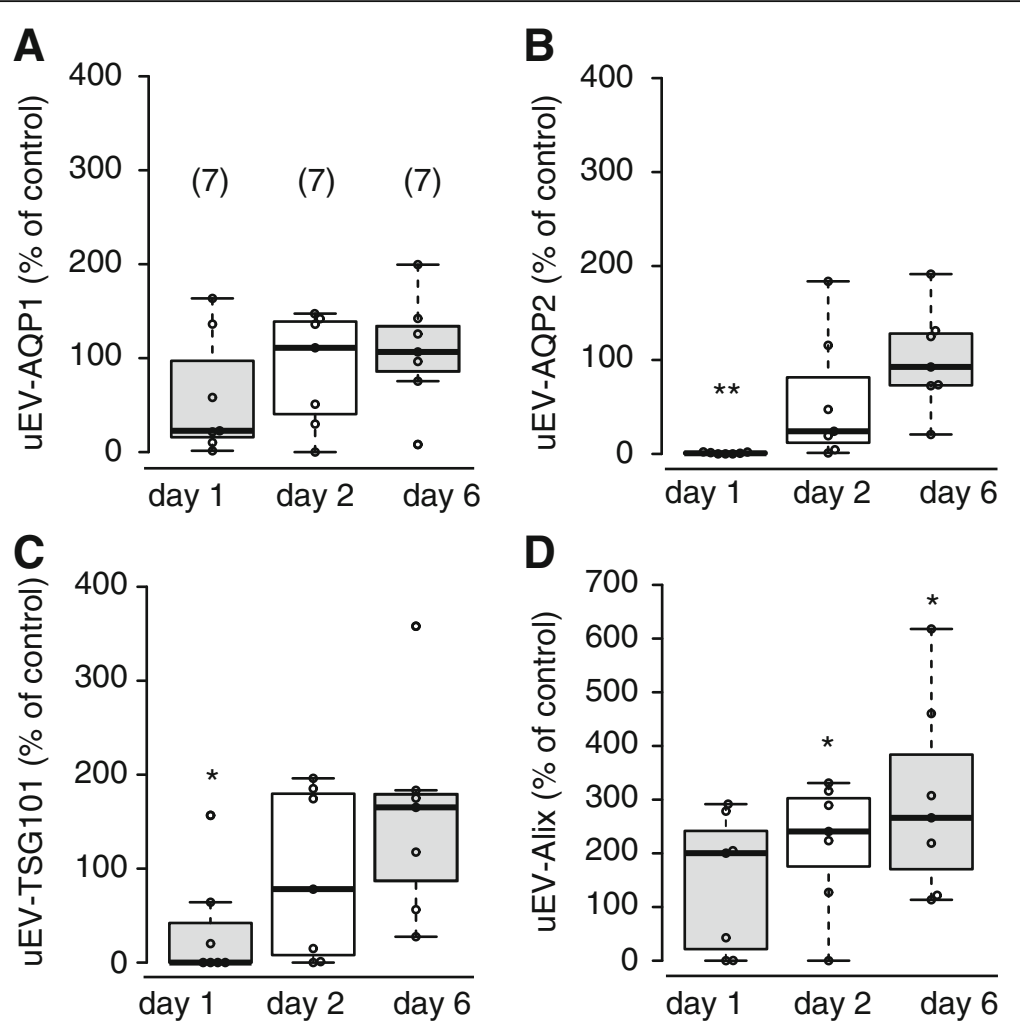

Fig. 3 Dot and box plots of uEV-AQP1, uEV-AQP2, uEV-TSG101, and uEV-Alix in recipients. Dot and box plots of release of uEV-AQP1 (a), uEVAQP2 (b), uEV-TSG101 (c), and uEV-Alix (d) on days 1, 2, and 6 in recipients. Each value is calculated as a percentage of the level of the control sample. ${ }^{*} P<0.01$ and ${ }^{*} P<0.05$, compared with the value of $100 \%$ (one sample t- test)

above the control level. On the other hand, the release of uEV-Alix was higher at all of the time points examined in this study relative to the control group. Although the reason for this discrepancy is currently unclear, one possibility may be that factors other than the number of exosomes released into urine affected the release of either uEV-TSG101 or -Alix. Since Alix is involved in cell death signaling as an apoptosis inducer [29], such signaling might be activated at all time points. In order to develop proteins in uEVs as a diagnosis tool, marker proteins in the uEVs would be important as an internal control for estimating the number of exosomes, and therefore further studies will be needed to clarify the internal control proteins that might be appropriate.
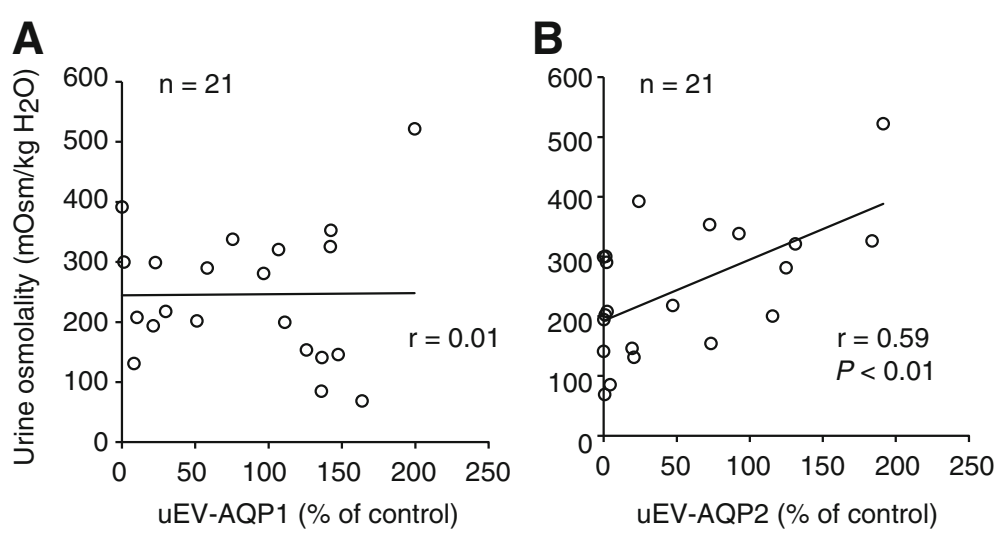

Fig. 4 Correlation between urinary osmolality and release of UEV-AQP1 or UEV-AQP2. The relationships between urinary osmolality and release of uEV-AQP1 (a) or -AQP2 (b) for all samples (on days 1, 2, and 6) are shown. The line is the least-squares regression line. $n$ and $r$ indicate the number of individuals and the correlation coefficient, respectively. There is a significant correlation between urinary osmolality and the release of uEV-AQP2, but not that of uEV-AQP1 (Pearson analysis, $P<0.01$ ) 
Our present findings suggest that uEV-AQP2 might be a useful marker for estimation of renal AQP2 dysregulation, thus affecting urinary concentration ability in recipients after renal transplantation. Recently, the beneficial effect of water intake has been discussed in terms of preservation of kidney function in patients with certain types of kidney disease through suppression of the plasma level of vasopressin [30]. Since AQP2 is a vasopressin-regulated water channel protein $[4,5]$, uEVAQP2 as a marker of renal water handling function might be useful for determining whether water intake therapy might be appropriate. Although direct measurement of blood vasopressin is problematic, as most of it (more than 90\%) binds to platelets and is unstable in collected plasma, it would be important in a future study to evaluate the relationship between uEV-AQP2 and plasma copeptin, which has been shown to be a useful surrogate marker of vasopressin [31].

\section{Conclusion}

This study has clearly provided information on the relationship between release of $\mathrm{uEV}-\mathrm{AQP} 2$ and the renal urinary concentration defect in the early phase after renal transplantation. However, this results were limited by the small sample size. Further studies with larger samples may provide more accurate information concerning the usefulness of this marker in renal transplantation.

\section{Abbreviations}

Alix: Apoptosis-linked gene 2-interacting protein X; AQP: Aquaporin; MVBs: Multivesicular bodies; TSG101: Tumor susceptibility gene 101: uEV: Urinary extracellular vesicle

\section{Acknowledgements}

None.

\section{Authors' contributions}

All authors have read and approved the final version. $\mathrm{SH}, \mathrm{NI}$, and $\mathrm{MI}$ designed the research, $\mathrm{SH}, \mathrm{NI}, \mathrm{HS}$, and $\mathrm{Ml}$ performed the research, $\mathrm{SH}, \mathrm{NI}, \mathrm{HS}$, and $\mathrm{MI}$ analyzed the data, $\mathrm{SH}$ and $\mathrm{MI}$ interpreted the results of experiments, $\mathrm{SH}$ and $\mathrm{MI}$ wrote the manuscript.

\section{Funding}

The work is supported by JSPS KAKENHI, 25660241 (M.I.), 25221205 (M.I.), $15 \mathrm{H} 04594$ (M.I.), 16 K15047 (M.I.), 24780287 (H.S.), and 15 K18784 (H.S.)

\section{Availability of data and materials}

The datasets for this study are available from M.I. on reasonable request.

\section{Ethics approval and consent to participate}

This study was approved by the Miyazaki Prefectural Miyazaki Hospital Institutional Review Board in accordance with the Ethical Guidelines for Clinical Studies in Japan (Miyazaki of Health, Labour and Welfare, July 30, 2003, Amended December 28, 2004). Written informed consent was obtained from all participants prior to their participation.

\section{Consent for publication}

Not applicable.

\section{Competing interests}

The authors declare that they have no competing interests.

\section{Author details}

${ }^{1}$ Department of Veterinary Pharmacology, Faculty of Agriculture, University of Miyazaki, Gakuenkibanadai-Nishi 1-1, Miyazaki 889-2192, Japan. Nephrology, Miyazaki Prefectural Miyazaki Hospital, Kitatakamatsu 5-30, Miyazaki 880-8510, Japan.

Received: 10 February 2018 Accepted: 28 May 2019

Published online: 11 June 2019

\section{References}

1. Ogden DA, Sitprija V, Holmes JH. Function of the renal homograft in man immediately after transplantation. Am J Med. 1965;38:873-82.

2. Henderson LW, Nolph KD, Puschett JB, Goldberg M. Proximal tubular malfunction as a mechanism for diuresis after renal homotransplantation. $N$ Engl J Med. 1968;278:467-73.

3. Preston GM, Carroll TP, Guggino WB, Agre P. Appearance of water channels in Xenopus oocytes expressing red cell CHIP28 protein. Science. 1992;256: 385-7.

4. Ikeda M, Matsuzaki T. Regulation of aquaporins by vasopressin in the kidney Vitam Horm. 2015:98:307-37.

5. Fushimi K, Uchida S, Hara Y, Hirata Y, Marumo F, Sasaki S. Cloning and expression of apical membrane water channel of rat kidney collecting tubule. Nature. 1993;361:549-52.

6. Colombo M, Raposo G, Théry C. Biogenesis, secretion, and intercellular interactions of exosomes and other extracellular vesicles. Annu Rev Cell Dev Biol. 2014:30:255-89.

7. Yáñez-Mó M, Siljander PR, Andreu Z, Zavec AB, Borràs FE, Buzas El, Buzas K, Casal E, Cappello F, Carvalho J, et al. Biological properties of extracellular vesicles and their physiological functions. J Extracell Vesicles. 2015;4:27066. https://doi.org/10.3402/jev.v4.27066.

8. Lötvall J, Hill AF, Hochberg F, Buzás El, Di Vizio D, Gardiner C, Gho YS, Kurochkin IV, Mathivanan S, Quesenberry P, et al. Minimal experimental requirements for definition of extracellular vesicles and their functions: a position statement from the International Society for Extracellular Vesicles. J Extracell Vesicles. 2014;3:26913. https://doi.org/10.3402/jev.v3.26913.

9. Karpman D, Ståhl AL, Arvidsson I. Extracellular vesicles in renal disease. Nat Rev Nephrol. 2017;13:545-62

10. Merchant ML, Rood IM, Deegens JKJ, Klein JB. Isolation and characterization of urinary extracellular vesicles: implications for biomarker discovery. Nat Rev Nephrol. 2017;13:731-49.

11. Pisitkun T, Shen RF, Knepper MA. Identification and proteomic profiling of exosomes in human urine. Proc Natl Acad Sci U S A. 2004;101:13368-73.

12. King HW, Michael MZ, Gleadle JM. Hypoxic enhancement of exosome release by breast cancer cells. BMC Cancer. 2012;12:421.

13. Garcia-Romero N, Esteban-Rubio S, Rackov G, Carrión-Navarro J, BeldaIniesta C, Ayuso-Sacido A. Extracellular vesicles compartment in liquid biopsies: Clinical application. Mol Asp Med. 2017;17:30103-6.

14. Ståhl AL, Johansson K, Mossberg M, Kahn R, Karpman D. Exosomes and microvesicles in normal physiology, pathophysiology, and renal diseases. Pediatr Nephrol. 2017;34:11-30.

15. Oshikawa $\mathrm{S}$, Sonoda $\mathrm{H}$, Ikeda M. Aquaporins in urinary extracellular vesicles (exosomes). Int J Mol Sci. 2016;17.

16. Sonoda H, Yokota-Ikeda N, Oshikawa S, Kanno Y, Yoshinaga K, Uchida K, Ueda Y, Kimiya K, Uezono S, Ueda A, et al. Decreased abundance of urinary exosomal aquaporin-1 in renal ischemia-reperfusion injury. Am J Physiol Renal Physiol. 2009;297:F1006-16.

17. Abdeen A, Sonoda H, El-Shawarby R, Takahashi S, Ikeda M. Urinary excretion pattern of exosomal aquaporin-2 in rats that received gentamicin. Am J Physiol Renal Physiol. 2014;307:F1227-F12237.

18. Velic A, Gabriëls G, Hirsch JR, Schröter R, Edemir B, Paasche S, Schlatter E. Acute rejection after rat renal transplantation leads to downregulation of $\mathrm{NA}+$ and water channels in the collecting duct. Am J Transplant. 2005;5: 1276-85.

19. Edemir B, Reuter S, Borgulya R, Schröter R, Neugebauer U, Gabriëls G, Schlatter $E$. Acute rejection modulates gene expression in the collecting duct. J Am Soc Nephrol. 2008;19:538-46.

20. Chen B, Zang CS, Zhang JZ, Wang WG, Wang JG, Zhou HL, Fu YW. The changes of aquaporin 2 in the graft of acute rejection rat renal transplantation model. Transplant Proc. 2010;42:1884-7.

21. Erdbrügger $U$, Le TH. Extracellular vesicles in renal diseases: more than novel biomarkers? J Am Soc Nephrol. 2016;27:12-26. 
22. Spitzer M, Wildenhain J, Rappsilber J, Tyers M. BoxPlotR: a web tool for generation of box plots. Nat Methods. 2014;11:121-2.

23. Kanda Y. Investigation of the freely available easy-to-use software 'EZR' for medical statistics. Bone Marrow Transplant. 2013;48:452-8.

24. Ren H, Yang B, Ruiz JA, Efe O, Ilori TO, Sands JM, Klein JD. Phosphatase inhibition increases AQP2 accumulation in the rat IMCD apical plasma membrane. Am J Physiol Renal Physiol. 2016;311:F1189-97.

25. Rinschen MM, Klokkers J, Pavenstädt H, Neugebauer U, Schlatter E, Edemir B. Different effects of CSA and FK506 on aquaporin-2 abundance in rat primary cultured collecting duct cells. Pflugers Arch. 2011;462:611-22.

26. Gong H, Wang W, Kwon TH, Jonassen T, Li C, Ring T, Frøkiær J, Nielsen S. EPO and alpha-MSH prevent ischemia/reperfusion-induced down-regulation of AQPs and sodium transporters in rat kidney. Kidney Int. 2004;66:683-95.

27. Kwon TH, Frøkiaer J, Fernández-Llama P, Knepper MA, Nielsen S. Reduced abundance of aquaporins in rats with bilateral ischemia-induced acute renal failure: prevention by alpha-MSH. Am J Phys. 1999;277:F413-27.

28. Ikeda M, Prachasilchai W, Burne-Taney MJ, Rabb H, Yokota-lkeda N. Ischemic acute tubular necrosis models and drug discovery: a focus on cellular inflammation. Drug Discov Today. 2006;11:364-70.

29. Sadoul R. Do Alix and ALG-2 really control endosomes for better or for worse? Biol Cell. 2006;98:69-77.

30. Wang CJ, Grantham JJ, Wetmore JB. The medicinal use of water in renal disease. Kidney Int. 2013;84:45-53.

31. Meijer E, Bakker SJ, de Jong PE, Homan van der Heide JJ, van Son WJ, Struck J, Lems SP, Gansevoort RT. Copeptin, a surrogate marker of vasopressin, is associated with accelerated renal function decline in renal transplant recipients. Transplantation. 2009;88:561-7.

\section{Publisher's Note}

Springer Nature remains neutral with regard to jurisdictional claims in published maps and institutional affiliations.

Ready to submit your research? Choose BMC and benefit from:

- fast, convenient online submission

- thorough peer review by experienced researchers in your field

- rapid publication on acceptance

- support for research data, including large and complex data types

- gold Open Access which fosters wider collaboration and increased citations

- maximum visibility for your research: over $100 \mathrm{M}$ website views per year

At BMC, research is always in progress.

Learn more biomedcentral.com/submissions 